\title{
Неуниверсальное скейлинговое поведение ширины пиков проводимости в режиме квантового эффекта Холла в структурах InGaAs/InAIAs
}

\author{
(C) С.В. Гудина ${ }^{1}$, Ю.Г. Арапов ${ }^{1}$, Е.В. Ильченко ${ }^{1}$, В.Н. Неверов ${ }^{1}$, А.П. Савельев ${ }^{1}$, С.М. Подгорных ${ }^{1,2}$, \\ Н.Г. Шелушинина ${ }^{1}$, М.В. Якунин ${ }^{1,2}$, И.С. Васильевский ${ }^{3}$, А.Н. Виниченко ${ }^{3}$ \\ ${ }^{1}$ Институт фризики металлов им. М.Н. Михеева Уральского отделения Российской академии наук, \\ 620108 Екатеринбург, Россия \\ ${ }^{2}$ Уральский федеральный университет им. Б.Н. Ельцина, \\ 620002 Екатеринбург, Россия \\ ${ }^{3}$ Национальный исследовательский ядерный университет „МИФИ“, \\ 115409 Москва, Россия \\ E-mail: svpopova@imp.uran.ru
}

(Получена 25 апреля 2018 г. Принята к печати 7 мая 2018 г.)

\begin{abstract}
Проведено исследование продольного $\rho_{x x}$ и холловского $\rho_{x y}$ сопротивлений в области квантовых фазовых переходов в режиме квантового эффекта Холла в магнитных полях до 12 Тл при температурах $T=0.4-30 \mathrm{~K}$ в двумерных электронных системах $n-\mathrm{In}_{0.9} \mathrm{Ga}_{0.1} \mathrm{As} / \mathrm{In}_{0.81} \mathrm{Al}_{0.19} \mathrm{As}$. Обнаружено неуниверсальное скейлинговое поведение температурной зависимости ширины пиков сопротивления $\rho_{x x}$, связанное с влиянием крупномасштабного случайного потенциала и смешиванием уровней Ландау с разными направлениями спина.
\end{abstract}

DOI: 10.21883/FTP.2018.12.46755.34

\section{1. Введение}

Критическое поведение проводимости вблизи переходов между локализованными-делокализованными состояниями в квантовом эффекте Холла является предметом неослабевающего интереса. Целочисленный квантовый эффект Холла $($ КЭХ) демонстрирует универсальное скейлинговое поведение [1-3] в рамках предположения о единственности критической точки (делокализованные состояния имеют одну энергию $E_{c}$ в центре уровней Ландау (УЛ)). Вблизи критической точки длина локализации $\xi$ расходится по степенному закону $\xi(E) \sim\left|E_{\mathrm{F}}-E_{c}\right|^{-\gamma}[1-3]$, где $E_{\mathrm{F}}-$ энергия Ферми и $\gamma$ - критический индекс длины локализации. При этом физические величины, такие как проводимость, следуют скейлинговому закону в зависимости от переменной $(L / \xi)^{1 / \gamma} \sim L^{1 / \gamma}\left|E_{\mathrm{F}}-E_{c}\right|, L-$ длина образца. В экспериментах, проводимых при конечных температурах, эффективной длиной образца для когерентного транспорта является длина $L_{\varphi} \sim T^{-p / 2}$, возникающая в результате эффектов сбоя фазы $[1,2]$ из-за неупругого электрон-электронного рассеяния, $T-$ температура, параметр $p$ - так называемый критический индекс длины неупругого рассеяния. Таким образом, скейлинговый параметр $\kappa$ для систем при конечной температуре имеет вид $\kappa=p / 2 \gamma[1-4]$.

Экспериментально было обнаружено, что температурные зависимости ширины $\Delta B$ пиков продольного сопротивления $\rho_{x x}$ и наклон $d \rho_{x y} / d B$ холловского сопротивления $\rho_{x y}$ при переходе между плато действительно демонстрируют степенной закон: $\Delta B \sim T^{\kappa}$ и $d \rho_{x y} / d B \sim T^{-\kappa}$. В экспериментальных работах [5-7] было установлено, что значение $\kappa$ является универсальным и равным 0.42 независимо от типа электронной системы и номера уровня Ландау, в целом ряде других экспериментов универсальность $\kappa$ была поставлена под сомнение [8-23,44].

Нерешенной проблемой при рассмотрении критических свойств проводимости в режиме КЭХ остается учет межэлектронных взаимодействий и спиновых эффектов, причем речь идет как о значении критических индексов, так и об особенностях локализации в присутствии смешивания УЛ, особенно для структур с сильным спин-орбитального взаимодействием. С одной стороны, на основе различных моделей и методов расчета был сделан вывод $[18,19]$, что при смешивании УЛ класс универсальности квантовых фазовых переходов не должен измениться. Однако существуют и работы, в которых даются весьма интересные предсказания о процессах локализации-делокализации носителей для близко расположенных УЛ [20,21,24]. В работах [20,21] показано, что около центра каждого уровня Ландау из-за межподзонного перемешивания будет формироваться узкая полоса делокализованных состояний и, как следствие, это приведет к нескейлинговому (non-scaling) поведению. Следует отметить, что до сих пор в центре внимания были в основном теоретические исследования. Есть лишь несколько экспериментов, где, в частности, исследовался один особый случай смешивания (mixing) УЛ с полностью вырожденными уровнями Ландау с противоположными ориентациями спина [25-28]. Таким образом, единая картина квантовых фазовых переходов при наличии смешивания УЛ с различными ориентациями спина в настоящее время отсутствует.

В нашей предыдущей работе [29] на системе InAlAs/InGaAs/InAlAs с высоким содержанием InAs не было обнаружено различия в значениях критических 
Конструкция и структурные параметры образцов InGaAs/InAlAs

\begin{tabular}{|c|c|c|}
\hline Слой & Состав слоя & Толщина слоя, (нм) \\
\hline 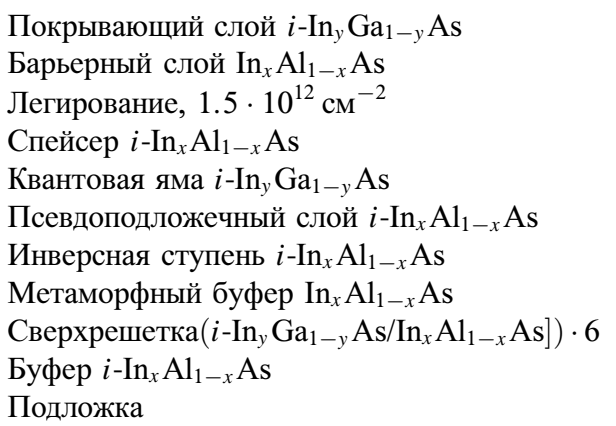 & $\begin{array}{c}y=0.90 \\
x=0.81 \\
\delta \text {-Si } \\
x=0.81 \\
y=0.90 \\
x=0.81 \\
x=0.83 \rightarrow 0.81 \\
x=0.55 \rightarrow 0.83 \\
y=0.53, x=0.52 \\
x=0.52 \\
(100) \text { InP }\end{array}$ & $\begin{array}{c}6 \\
96 \\
\\
12 \\
14 \\
140 \\
19 \\
1350 \\
78 \\
42\end{array}$ \\
\hline
\end{tabular}

индексов для спин-расщепленных и спин-вырожденных УЛ. В данной работе мы продолжили поиски на подобных структурах с более высокой концентрацией и подвижностью электронов.

\section{2. Образцы и методика эксперимента}

Полупроводниковые гетероструктуры с квантовой ямой $\operatorname{In}_{0.81} \mathrm{Al}_{0.19} \mathrm{As} / \mathrm{In}_{0.9} \mathrm{Ga}_{0.1} \mathrm{As} / \mathrm{In}_{0.81} \mathrm{Al}_{0.19} \mathrm{As} \quad$ и метаморфным буфером изменяющегося состава $\mathrm{In}_{x} \mathrm{Al}_{1-x} \mathrm{As}$ были выращены на установке RIBER Compact 21 в Институте функциональной ядерной электроники Национального исследовательского ядерного университета „МИФИ“ методом молекулярно-лучевой эпитаксии. Для роста использовались подложки InP (Wafer Technology Co.) с ориентацией (100). Последовательность и параметры слоев в образце представлены в таблице. Концентрация электронов $n=6.9 \cdot 10^{15} \mathrm{M}^{-2}$, подвижность $\mu=20 \mathrm{M}^{2} / \mathrm{B} \cdot \mathrm{c}$.

Профиль состава (концентрация индия $x$ ) в метаморфном буфере $\operatorname{In}_{x} \mathrm{Al}_{1-x}$ As был технологически задан линейным. Для подавления прорастания дислокаций в область квантовой ямы в структуру была введена сверхрешетка. Метаморфный буфер завершался инверсной ступенью с линейно уменьшающейся концентрацией, чтобы предотвратить проникновение упругих напряжений в активную область. Затем выращивался толстый слой псевдоподложки $\operatorname{In}_{x} \mathrm{Al}_{1-x} \mathrm{As}$ в качестве подложки для последующих активных слоев квантовой ямы $\mathrm{In}_{y} \mathrm{Ga}_{1-y} \mathrm{As}$, затем следовали спейсер $\mathrm{In}_{x} \mathrm{Al}_{1-x} \mathrm{As}, \delta$-слой легирующей примеси $\mathrm{Si}$, барьерный слой $\operatorname{In}_{x} \mathrm{Al}_{1-x} \mathrm{As}$ и нелегированный защитный слой $\operatorname{In}_{y} \mathrm{Ga}_{1-y} \mathrm{As}$. Образец на подложках InP содержал псевдоморфно-напряженную квантовую яму, чей параметр решетки превышал параметр решетки барьерных слоев. Кремний использовался в качестве донорной примеси, концентрация атомов $1.5 \cdot 10^{12} \mathrm{~cm}^{-2}$. Дополнительную информацию о технических условиях роста можно найти в работах [30,31].

Образец был изготовлен в форме двойного холловского мостика, ширина канала $w=0.6$ мм, отношение длины к ширине $L / w=3.5$. Продольное $\rho_{x x}$ и холловское $\rho_{x y}$ магнитосопротивления измерялись на постоянном токе $I=1$ мкА одновременно в магнитных полях до 9.0 Тл при температуре от 1.8 до $30.0 \mathrm{~K}$ на установке PPMS Quantum Design, а также в магнитных полях до 12 Тл при температуре от 0.4 до $1.6 \mathrm{~K}$ с использованием вставки $\mathrm{He}^{3}$ на установке Oxford Instruments.

\section{3. Результаты и обсуждение}

\section{1. Результаты эксперимента}

На рис. 1 представлены магнитополевые зависимости продольной и холловской компонент тензора сопротивления для исследованного образца в перпендикулярных магнитных полях до $B=12$ Тл при $T=1.5 \mathrm{~K}$. На зависимостях $\rho_{x y}(B)$ хорошо видны квантованные плато $\rho_{x y}=v h / e^{2}$ для четных и нечетных факторов заполнения $v=3,4,5,6$ и т. д. до $v=20$, на $\rho_{x x}(B)-$ хорошо выраженные осцилляции Шубникова-де Гааза. На вставке к рис. 1 показаны типичные зависимости $\rho_{x x}(B)$ при фиксированных температурах.

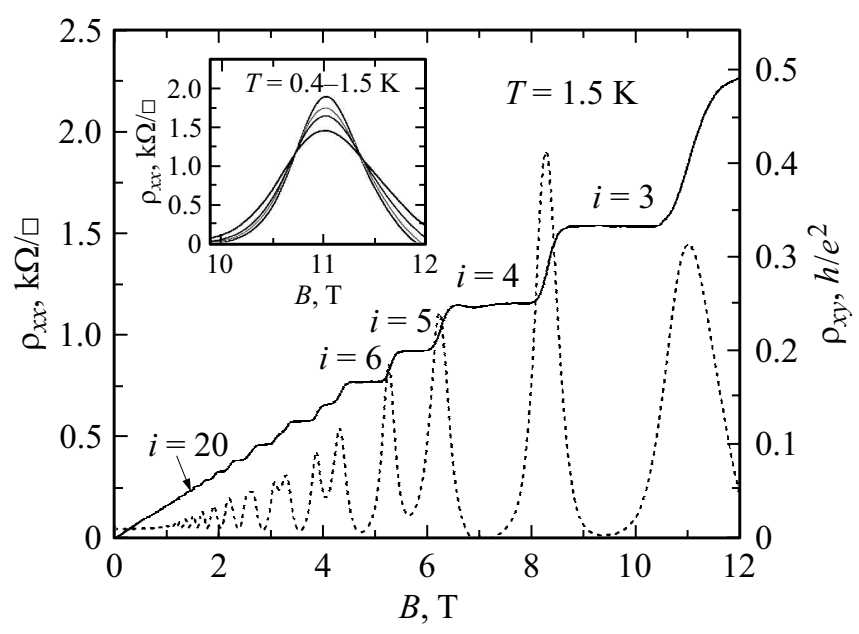

Рис. 1. Магнитополевые зависимости $\rho_{x x}$ и $\rho_{x y}$ при температуpe $T=1.5 \mathrm{~K}$. На вставке - зависимости $\rho_{x x}(B)$ для перехода $2 \rightarrow 3$ при фиксированных температурах $0.4-1.5 \mathrm{~K}$. 

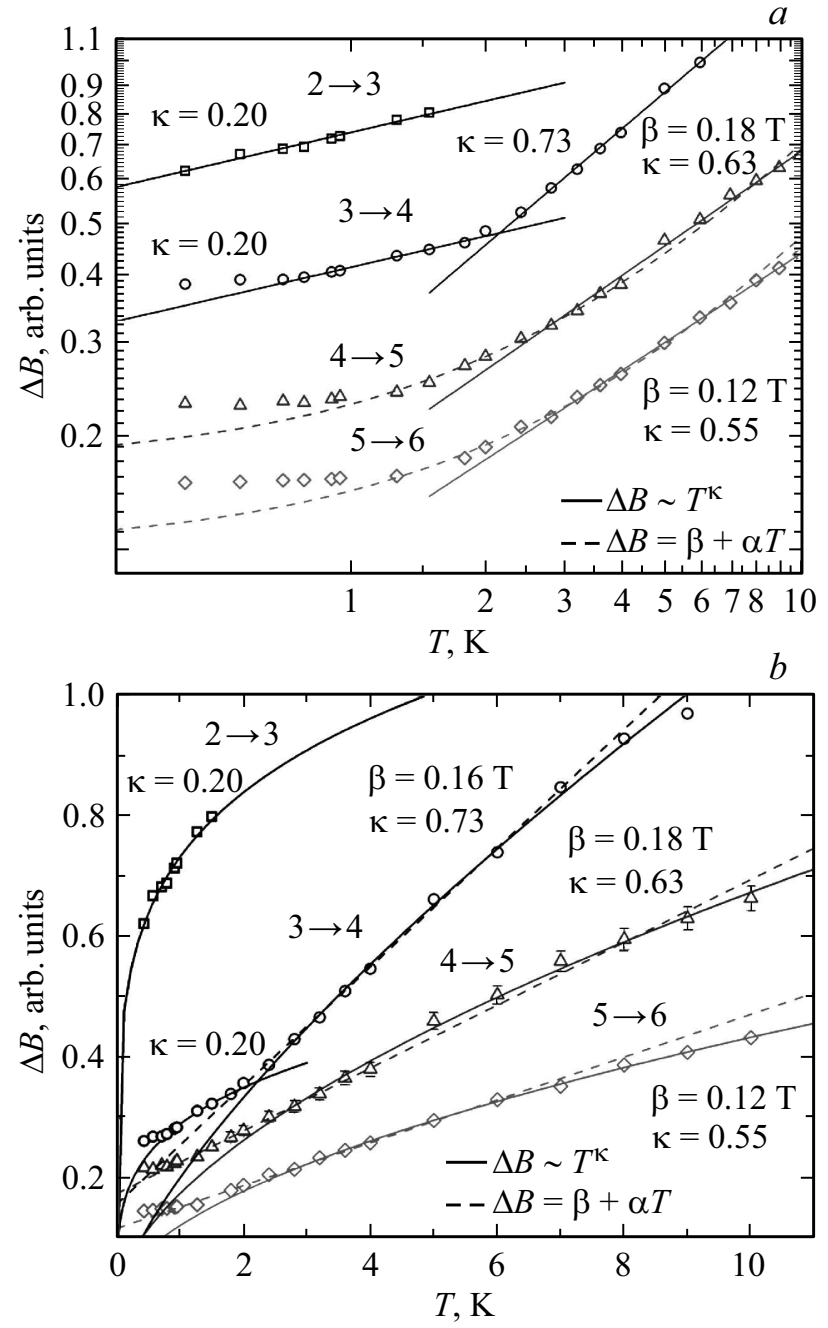

Рис. 2. Температурные зависимости ширины $\Delta(B)$ пиков $\rho_{x x}$, для переходов между плато $2 \rightarrow 3,3 \rightarrow 4,4 \rightarrow 5$ и $5 \rightarrow 6$ (сдвинуты для наглядности) в двойном логарифмическом масштабе $(a)$ и в линейном масштабе $(b)$. Сплошные линии $\Delta B \propto T^{\kappa}$, пунктирные линии $-\Delta B \propto \beta+\alpha T$.

На рис. 2 показаны температурные зависимости ширины $\Delta B(T)$ пиков $\rho_{x x}(\mathrm{~B})$, определенные как расстояние между двумя экстремумами $d \rho_{x x} / d B . \Delta B$ является мерой доли делокализованных состояний на уровне Ландау, участвующих в проводимости при конечных температуpax. Видно (рис. 2, $a$ ), что $\Delta B(T)$ при $T \rightarrow 0$ уменьшается по степенному закону $T^{\kappa}$, причем для переходов между плато с разными факторами заполнения значения критических индексов при $T>2 \mathrm{~K}$ отличаются. Можно выделить три температурные области, в которых скорость изменения ширины перехода между плато КЭХ при изменении температуры различна: 1) наблюдается скейлинговое поведение $\Delta B \sim T^{\kappa}$ с критическим индексом $\kappa=[(0.55-0.73) \pm 0.02]$ при $T>2 \mathrm{~K}$ для переходов $3 \rightarrow 4,4 \rightarrow 5,5 \rightarrow 6$; 2) $\kappa=[0.20 \pm 0.02]$ при $T<2 \mathrm{~K}$ для $2 \rightarrow 3$ и $3 \rightarrow 4$; 3$)$ при $T<0.9 \mathrm{~K}$ для переходов $3 \rightarrow 4,4 \rightarrow 5,5 \rightarrow 6$ зависимость $\Delta B$ стремится к на- сыщению. Для переходов $4 \rightarrow 5$ и $5 \rightarrow 6$ при $T<5 \mathrm{~K}$ зависимости $\Delta B(T)$ лучше описываются линейным законом $\Delta B(T)=\alpha T+\beta$.

Начиная с пионерского экспериментального исследования на низкоподвижных гетероструктурах InGaAs/InP [5], где был подтвержден степенной закон поведения $\Delta B(T)$ и $d \rho_{x y} /\left.d B\right|_{B_{c}}(T)$ для нескольких уровней Ландау, значение $\kappa=0.42 \pm 0.04$ считается универсальным. Скейлинговое поведение с $\kappa=0.42 \pm 0.01$ было обнаружено и для переходов плато - плато КЭХ в гетероструктурах $\mathrm{Al}_{x} \mathrm{Ga}_{1-x} \mathrm{As} / \mathrm{Al}_{0.33} \mathrm{Ga}_{0.67} \mathrm{As}$ в области сплавного рассеяния [6,7]. Недавние эксперименты на структурах $n$ - $\operatorname{In}_{0.2} \mathrm{Ga}_{0.8} \mathrm{As} / \mathrm{GaAs} \quad$ с двойными квантовыми ямами выявили реальное скейлинговое поведение с $\kappa=0.48 \pm 0.04$ для переходов между плато $1 \rightarrow 2$, что авторы связывают с решающей ролью мелкомасштабного примесного потенциала [32]. Значения $\kappa=0.42$ были обнаружены для первого и второго уровней Ландау (как для электронов, так и для дырок) в монослое графена [33]. Последние результаты по КЭХ в структурах на основе $\mathrm{HgTe}$ с квантовыми ямами шириной 5.9 нм для переходов $1 \rightarrow 2,2 \rightarrow 3$ также показывают значения $\kappa=0.45 \pm 0.04$ в интервале температур 0.4-50 K [34].

Однако в других экспериментальных работах универсальность критического индекса $\kappa$ была поставлена под сомнение. Основными причинами такой неуниверсальности называются влияние крупномасштабного примесного потенциала $[3,6,7,35,36]$, макронеоднородности в образце (в частности, неоднородности распределения концентрации носителей заряда по образцу) [3,37], межэлектронные взаимодействия и спиновые эффекты $[3,38,39]$.

\section{2. Влияние крупномасштабного случайного потенциала}

Значения $\kappa$ при $T>2 \mathrm{~K}$, определенные в настоящем исследовании, хорошо согласуются со значениями, получаемыми на системах с крупномасштабным случайным потенциалом $[6,7,11,15,28,29,33,35,36,40-44]$. В частности, во множестве работ для наиболее изученной структуры GaAs/AlGaAs регулярно наблюдаются $\kappa=0.5-0.75$.

Так, при исследованиях перехода плато КЭХ (с номером $i=1)-$ холловский изолятор для структур с квантовыми ямами $\operatorname{In}_{0.53} \mathrm{Ga}_{0.47} \mathrm{As} / \mathrm{InP}$ и $\mathrm{GaAs} / \mathrm{AlGaAs}$ выявлено скейлинговое поведение с $\kappa=0.56 \pm 0.02$ ([3] и ссылки там). Показана необходимость учета макроскопических неоднородностей в образце и важность исследования переходов плато-холловский изолятор по сравнению с переходами плато-плато КЭХ, так как это дает возможность ввести так называемые поправки к скейлингу [3]. Причем такие факторы, как крупномасштабный случайный потенциал и наличие макронеоднородностей, являются конкурирующими: крупномасштабный потенциал увеличивает значение $\kappa$, в то время как присутствие неоднородностей уменьшает $\kappa$ [3]. 
В работах $[6,7,36]$ наблюдение зависимости значения $\kappa$ от концентрации $\mathrm{Al}(x)$ в квантовой яме $\mathrm{Al}_{x} \mathrm{Ga}_{1-x} \mathrm{As} / \mathrm{Al}_{0.33} \mathrm{Ga}_{0.67} \mathrm{As}$ явилось ярким доказательством влияния масштаба случайного потенциала на значение $\kappa$. Изменение $\kappa$ от 0.56 до 0.42 и вновь до 0.56 при изменении $x$ от 0 до 0.0065 и до 0.016 , сопровождаемое изменением масштаба случайного потенциала [45], трактуется авторами как смена процессов классического протекания (крупномасштабный потенциал) процессами квантового туннелирования (короткодействующий случайный потенциал) и обратно с ростом степени беспорядка при изменении содержания алюминия в активном слое квантовой ямы.

Недавно была исследована температурная зависимость ширины области перехода между плато КЭХ в квантовых ямах $\mathrm{HgTe}$ с инвертированным зонным спектром [40]. Было обнаружено скейлинговое поведение с критическим индексом $\kappa=0.54 \pm 0.01$ на переходе $1 \rightarrow 2$ в широком интервале температур $T=2.9-30 \mathrm{~K}[40]$. Этот результат объяснен на основе того факта, что основной причиной рассеяния электронов в исследованной структуре являлась примесь In, отделенная спейсером от двумерного слоя, так что в системе имелся крупномасштабный случайный потенциал.

Крупномасштабный (медленно меняющийся) потенциал считается основным типом беспорядка в стандартных гетероструктурах на основе GaAs, что исторически привело к полуклассическому рассмотрению (перколяционной картине) эффектов делокализации вблизи центра размытого уровня Ландау (см. ссылки в работе [2]).

В работе [4], посвященной протеканию и квантовому туннелированию в режиме КЭХ, была предложена модель регулярной сетки, которая сделала возможным численные расчеты систем с медленно меняющимся на масштабе магнитной длины беспорядком и включила в рассмотрение эффекты интерференции и квантового туннелирования, авторы работы [4] получили значение $\gamma=2.5 \pm 0.5$, в разумном согласии со значениями для быстро меняющегося (короткодействующего) потенциала [2]. В классической картине длина локализации экспоненциально расходится с показателем $\gamma=4 / 3$ [4].

В работе [46] предложен способ оценки ширины полосы квантового протекания, т.е. интервала энергий $W_{0}$, где сохраняется квантовая фазовая когерентность, по отношению к полной ширине $W$ уровня Ландау. Предполагая простую квадратичную форму потенциала вблизи седловой точки, было получено выражение $W_{0} \approx\left(l_{B} / a\right)^{2} W$, где $l_{B}-$ магнитная длина, $a-$ характерная корреляционная длина случайного потенциала, которая должна быть много больше $l_{B}$ и $W$ равно амплитуде флуктуаций потенциала. Эта оценка означает, что для разных образцов и разных номеров УЛ ширина полосы $W_{0}$, где наблюдается так называемый истинный скейлинг, различна, а значит, и условия ее достижения различны. Для классического протекания $\gamma=4 / 3$, тогда, зная $\kappa=p / 2 \gamma$ и $p=2$, получаем $\kappa=0.75$, значение, ре- гулярно получаемое в системах с медленно меняющимся потенциалом.

Таким образом, значения $\kappa$, полученные в наших экспериментах при $T>2 \mathrm{~K}$, связаны с присутствием в образце крупномасштабного случайного потенциала, сформированного различными причинами: удаленное легирование, особенности конструкции образца, например, дислокации, возникающие в метаморфном буфере [30].

Разные значения $\kappa$ для переходов между плато КЭХ с разными номерами получены в систематических исследованиях серий образцов $\mathrm{GaAs} / \mathrm{Al}_{x} \mathrm{Ga}_{1-x} \mathrm{As}[11,22]$. В работе [22] показано, что, как и в настоящей работе, $\kappa$ уменьшается с увеличением номера уровня Ландау и с уменьшением концентрации, изменяемой в пределах от 2.2 до $5.9 \cdot 10^{11} \mathrm{~cm}^{-2}$ с помощью напряжения на затворе.

\section{3. Влияние межэлектронного взаимодействия и спиновых эффектов}

Значения $\kappa=0.20 \pm 0.02$, полученные при $T<2 \mathrm{~K}$, наблюдались и ранее. Впервые $\kappa=0.21$ было обнаружено для спин-вырожденных уровней Ландау (переход $2 \rightarrow 4$ в $\operatorname{In}_{0.53} \mathrm{Ga}_{0.47} \mathrm{As} / \mathrm{InP}$ [25]). Если вспомнить, что $\kappa=p / 2 \gamma$ есть комбинация двух микроскопических параметров, а именно $p$, который определяет температурную зависимость времени неупругого рассеяния, $\tau_{\text {in }} \propto T^{-p}$, и $\gamma$, определяющего скорость расходимости длины локализации при приближении к критической точке, то возникает вопрос (см. работу[25]), на какой критический индекс, $p$ или $\gamma$, влияет спиновое вырождение.

Значения $\kappa=0.21 \pm 0.01$ для спин-поляризованных уровней Ландау для переходов $2 \rightarrow 3$ и $3 \rightarrow 4$ были получены в гетероструктурах $n$ - $\mathrm{In}_{0.2} \mathrm{Ga}_{0.8} \mathrm{As} / \mathrm{GaAs}$ с двойными квантовыми ямами с максимальной концентрацией электронов, достигнутой путем подсветки инфракрасным излучением, и объяснены влиянием электронэлектронного взаимодействия конечного радиуса, связанного с эффективным экранированием дальнодействующего потенциала кулоновского электрон-электронного взаимодействия в одной квантовой яме с помощью другой [32].

В работе [23] на структурах $\mathrm{AlGaAs} / \mathrm{GaAs}$ анализировалось изменение ширины пиков $\Delta B$ проводимости $\rho_{x x}$ в режиме КЭХ с температурой $\Delta B \sim T^{\kappa}$ и частотой $\Delta B \sim f^{\kappa}$. Неуниверсальное поведение $(\kappa>0.4)$, связанное с примесями, было отделено от универсального скейлинга $(\kappa \approx 0.4)$. При сравнении спин-расщепленных $(\kappa \sim 0.4)$ и спин-вырожденных $(\kappa \sim 0.2)$ пиков $\sigma_{x x}$ была продемонстрирована важность учета обменного взаимодействия и усиления $g$-фактора.

Кроме того, интересным является тот факт, что значения критических индексов $\kappa \cong(0.2-0.3)$ регулярно наблюдаются на различных типах двумерных структур на основе графена ([47] и ссылки в ней). Такое неуниверсальное поведение, по мнению исследователей графена, свидетельствует о протекании в присутствии 
крупномасштабного случайного потенциала, в противовес традиционным полупроводниковым 2D системам, рассмотренным в разд. 3.2 настоящей статьи.

Обсудим предсказания теории относительно значений критических индексов. Теоретическая концепция [1] относится к системам невзаимодействующих электронов, в то время как в реальных системах требуется учет электрон-электронных $(e-e-)$ взаимодействий. Попытки учета $e-e$-взаимодействий при рассмотрении критических свойств для переходов плато-плато КЭХ предпринимались постоянно (см., например, [48]), но последовательный подход был развит в работах $[38,39,49]$ и систематизирован в [3]. Обобщенный подход [3] согласует механизм делокализации в сильном квантующем магнитном поле с теорией Финкельштейна для явлений локализации и $e-e$-взаимодействия [50]. Этот подход оправдывает применимость концепции скейлинга для анализа ширины переходов между плато КЭХ для взаимодействующих электронов (трехпараметрический скейлинг, в качестве третьего безразмерного параметра принимается константа взаимодействия в триплетном канале). В частности, в случае короткодействующего потенциала $e-e$-взаимодействия фиксированная точка, соответствующая делокализованному состоянию $\sigma_{x y}=i+1 / 2$, остается стабильной по отношению к взаимодействию $[3,38,48,49]$.

Теории двухпараметрического скейлинга (для невзаимодействующих электронов) [1] или трехпараметрического скейлинга (с учетом $e$-e-взаимодействия) [3], хотя и решают фундаментальные проблемы, но не дают конкретных значений критических индексов. Результаты численных расчетов критического индекса длины локализации для модели невзаимодействующих электронов, полученные во многих последующих исследованиях (см. обзоры $[2,3]$ ), дают значение $\gamma=2.35 \pm 0.03$. Принимая $\kappa=p / 2 \gamma$ и $p=2$, немедленно получаем классическое и считающееся универсальным значение $\kappa=0.42$. Количественные оценки предполагаемого значения параметра $\kappa$ в моделях с короткодействующим $e-e$-взаимодействием следующие: $\kappa=0.21(\gamma=2.3$ и $z=2$ - динамический критический индекс, $\kappa=1 / z \gamma)$ [48]; $\kappa=0.21$ $(\gamma=2.3$ и $p=1) \quad[49] ; \kappa=0.27 \pm 0.04 \quad(\gamma=2.75$ и $p=1.35 \pm 0.15)[3,38]$.

Принимая во внимание существующие предсказания теории и результаты предыдущих экспериментов, можно сделать два предположения о природе низкотемпературных $(T<2 \mathrm{~K})$ значений $\kappa$ в изучаемой системе: 1) экспериментально извлеченные $\kappa$ хорошо согласуются с теоретически предсказываемыми для систем с малым радиусом $e-e$-взаимодействия; 2) мы не можем игнорировать влияние неоднородностей.

\section{4. Переход от неуниверсального скейлинга к универсальному}

Работы, в которых наблюдается переход от высокотемпературного, неуниверсального режима скейлин- га к низкотемпературному, универсальному, единичны. Первой следует назвать одну из пионерских работ [35], где кроссовер от нескейлингового поведения к скейлинговому с $\kappa=0.42$ наблюдался для серии гетероструктур $\mathrm{GaAs} / \mathrm{AlGaAs}$ из-за доминирования крупномасштабных флуктуаций случайного потенциала. В работе [6] представлены экспериментальные данные по переходам плато-плато КЭХ в гетероструктурах $\mathrm{Al}_{x} \mathrm{Ga}_{1-x} \mathrm{As} / \mathrm{Al}_{0.32} \mathrm{Ga}_{0.68} \mathrm{As}$ (c $x=0$ и $0.21 \%$ ) в диапазоне температур от $1.2 \mathrm{~K}$ до $1 \mathrm{мK}$, где наблюдался кроссовер от неуниверсального режима $(\kappa=0.58)$ при высоких температурах к универсальному $(\kappa=0.42)$ при низких температурах. Температура кроссовера увеличивалась с ростом $x$ от $120 \mathrm{MK}$ при $x=0 \%$ до $250 \mathrm{мК} \mathrm{при}$ $x=0.21 \%$ благодаря увеличению вклада в беспорядок от короткодействующего по природе сплавного рассеяния. Когда концентрация Al достигает $x=0.85 \%$, тогда беспорядок полностью определяется короткодействующим случайным примесным потенциалом, температура кроссовера $T_{\text {cross }}$ превышает $1.2 \mathrm{~K}$ и универсальный скейлинг $(\kappa=0.42)$ наблюдается во всем исследованном диапазоне температур.

Это поведение интерпретируется в работе [6] как переход от процессов теплового возбуждения через потенциальный барьер в седловых точках при $T>T_{\text {cross }}$ к процессам квантового туннелирования при $T<T_{\text {cross. }}$ Универсальный скейлинг с критическим индексом $\kappa=0.42$ становится наблюдаемым только при достаточно низких температурах, когда при $T<T_{\text {cross }}$ длина фазовой когерентности превышает типичный размер флуктуаций примесного потенциала и доминируют процессы когерентного туннелирования.

В работе [51] мы наблюдали кроссовер из неуниверсального скейлингового режима при $T>2 \mathrm{~K}$ с $\kappa=0.70 \pm 0.12 \quad$ к универсальному при $T<2 \mathrm{~K} \quad$ с $\kappa=0.25 \pm 0.02$ в структурах $n$ - $\operatorname{In}_{0.2} \mathrm{Ga}_{0.8} \mathrm{As} / \mathrm{GaAs}$ с одиночной квантовой ямой с максимальной концентрацией электронов, полученной с помощью ИК-подсветки.

Значения $\kappa$ с точностью до погрешности измерения, как до кроссовера, а также после него [51], хорошо согласуются со значениями, полученными в настоящем исследовании. Совпадает также и температура $T_{\text {cross }}=2 \mathrm{~K}$. Можно предположить случайное совпадение объема вклада в потенциал беспорядка от короткодействующего сплавного рассеяния в двух разных по составу, конструкции и свойствам 2D электронов гетероструктурах на основе InGaAs, который, как показано в работе [6], определяет как $\kappa$, так и $T_{\text {cross }}$.

\section{5. Насыщение температурной зависимости ширины перехода $\Delta B(T)$}

Насыщение, обнаруженное на переходах $3 \rightarrow 4,4 \rightarrow 5$, $5 \rightarrow 6$ при $T<0.9 \mathrm{~K}$, наблюдалось и ранее $[6,7,10,11]$ и связано с размерными эффектами, а именно с ограничением режима скейлинга в пределе низких температур, когда длина фазовой когерентности сравнивается с 
характерным размером образца. Так, в работе [7] было показано, что температура насыщения растет с 0.06 до $0.2 \mathrm{~K}$ при уменьшении размера образца с 500 до 100 мкм. При этом необходимо следить, чтобы были исключены нелинейные эффекты, связанные с разогревом электронного газа за счет величины тока, пропускаемого через образец. У нас насыщение происходит при высокой температуре $T \cong 0.9 \mathrm{~K}$ при достаточно больших размерах образца (наименьший характерный размер это ширина токового канала на холловском мостике 600 мкм). С другой стороны, эксперименты показали [52], что разогрев 2D электронного газа пропускаемым током начинается при $I>4$ мкА при температуре образца $T=0.4 \mathrm{~K}$. Таким образом, вопрос о насыщении требует дополнительного исследования.

\section{6. Линейная температурная зависимость ширины перехода $\Delta B(T)$}

Обсудим теперь линейный ход зависимости $\Delta B(T)$, обнаруженный для переходов между плато КЭХ с большими номерами. На рис. $2, b$ зависимости $\Delta B(T)$ представлены в линейном масштабе. Видно, что при $T<3$ К для переходов $4 \rightarrow 5$ и $5 \rightarrow 6$ данные не могут быть удовлетворительно описаны степенным законом $\Delta B \sim T^{\kappa}$. С другой стороны, данные хорошо описываются линейной зависимостью $\Delta B(T)=\alpha T+\beta$ с параметрами $\alpha=0.055$ Тл/К, $\beta=0.18$ Тл для перехода $4 \rightarrow 5, \alpha=0.036$ Тл/K, $\beta=0.12$ Тл для перехода $5 \rightarrow 6$. В температурном интервале от 3 до 6-9 К как степенной, так и линейный законы вполне хорошо подходят для описания экспериментальных данных. Отклонение между двумя подгонками лежит в пределах экспериментальных погрешностей (см. данные для перехода $4 \rightarrow 5$ на рис. $2, b)$.

В работах [12,13] сообщалось о новом транспортном режиме, отличающемся от критического скейлингового поведения, который существует асимптотически близко к переходу при очень низких температурах. Изучая переход „КЭХ-диэлектрик“ в образцах различных структур $\mathrm{GaAs} / \mathrm{AlGaAs}$ и InGaAs/InP при температурах до 70 мК, была обнаружена экспоненциальная зависимость $\rho_{x x}$ по обе стороны от значения критического фактора заполнения $v_{c}\left(\Delta v=\left|v-v_{c}\right|\right), \rho_{x x}=\exp \left(-\Delta v / v_{0}(T)\right)$, и отмечено, что эффективная ширина перехода $v_{0}(T)$ изменяется как $\alpha T+\beta$, вместо $T^{\kappa}$ для скейлингового поведения. Это означает, что даже при $T=0$ переход имеет конечную ширину и другой механизм проводимости при более низких температурах. Авторы отметили, что некоторые из их образцов на основе InGaAs/InP были из той же серии, что и в работе [5], и что они также пересмотрели свои предыдущие данные для образцов $\mathrm{GaAs} / \mathrm{AlGaAs}$ в работах $[12,13]$.

Какова причина появления линейной зависимости $\Delta B(T)=\alpha T+\beta$ ? Самой простой и естественной причиной для линейной зависимости $\Delta B(T)$ является температурное уширение квантового критического фазового пе- рехода. Она была предложена и подтверждена расчетами в работе [14]. Было показано, что температурное уширение функции распределения Ферми-Дирака $f(E)$ приводит к линейной температурной зависимости $\Delta B(T)$. Однако такое поведение должно предшествовать скейлинговым закономерностям при уменьшении температуры, как показано, например, в работах [14 и ссылки в ней]. В работах $[12,17]$, как и в настоящей, линейная зависимость наблюдается вплоть до самых низких температур.

В теоретической работе [46] и в экспериментальной работе [13] было отмечено очень важное значение короткодействующего случайного потенциала рассеяния для изучения скейлинговых явлений, так как дальнодействующий потенциал рассеяния резко усложняет наблюдаемость критических явлений. По их мнению, линейный ход $(\Delta B(T)=\alpha T+\beta)$ является квазиклассическим по своему характеру и должен наблюдаться при конечных $T$ и на образцах с преобладанием медленно меняющихся флуктуаций потенциала беспорядка.

Авторы работы [16] считают, что ответ на вопрос о конечной ширине переходов КЭХ при $T \rightarrow 0$ может быть найден при учете влияния кулоновских электронэлектронных взаимодействий на экранирование плавного потенциала беспорядка $[54,55]$. Эта теория рассматривает экранирование в рамках приближения Томаса-Ферми, которое подходит для плавного потенциала беспорядка. Когда имеет место смешивание уровней Ландау, взаимодействие электронов на разных уровнях Ландау с одинаковыми номерами и противоположно направленными спинами серьезно усложняет эту проблему, особенно в случае структур с сильным спин-орбитальным взаимодействием, каковыми являются структуры на основе InGaAs с высоким содержанием InAs.

Кроме того, возникает еще один вопрос, изменит ли смешивание уровней Ландау закон универсальности скейлингового поведения. В целом ряде теоретических работ был сделан вывод о том, что смешивание уровней Ландау не меняет класс универсальности при квантовых фазовых переходах в режиме КЭХ $[17,18]$. Однако в работе [20] было убедительно показано, что узкие металлические области образуются около каждого центра УЛ из-за их смешивания. Следует отметить, что в работе [21] теория была развита дальше путем включения в рассмотрение межзонных перекрытий. Было показано, что только для очень чистых образцов делокализованные состояния на каждом уровне Ландау остаются в одной критической точке при нулевой температуре, в отличие от достаточно грязных образцов, в которых делокализованные состояния на каждом уровне Ландау могут сформировать узкую полосу вместо одной критической точки. Кроме того, в [21] показано, что при определенной степени беспорядка возможен режим, когда для разных номеров УЛ могут реализовываться противоположные ситуации: для малых номеров УЛ при нулевой температуре остается только одно делокализованное состояние в критической точке, в то время как для больших номеров УЛ могут быть сформированы узкие полосы состояний 
конечной ширины, как раз то, что мы наблюдаем в этой работе. Таким образом, наш эксперимент может свидетельствовать о наличии полосы делокализованных состояний, являющейся следствием сильного смешивания и перекрытия уровней Ландау с одинаковыми номерами и противоположно направленными спинами.

\section{4. Заключение}

Проведены измерения температурных зависимостей продольной $\rho_{x x}$ и холловской $\rho_{x y}$ компонент тензора сопротивления в режиме квантового эффекта Холла в двумерных электронных системах $n$ - $\operatorname{In}_{0.9} \mathrm{Ga}_{0.1} \mathrm{As} / \mathrm{In}_{0.81} \mathrm{Al}_{0.19} \mathrm{As}$. В рамках концепции скейлинга проведен анализ температурных зависимостей ширины пиков $\rho_{x x}(B)$ для переходов между плато КЭХ $2 \rightarrow 3,3 \rightarrow 4,4 \rightarrow 5$ и $5 \rightarrow 6$.

Обнаружено, что для переходов $2 \rightarrow 3,3 \rightarrow 4$ при $T<2 \mathrm{~K}$ наблюдается скейлинговое поведение $\Delta B \sim T^{\kappa}$ с критическим индексом $\kappa=0.20 \pm 0.02$, предсказываемым теорией для систем с короткодействующим электрон-электронным взаимодействием.

Для перехода $3 \rightarrow 4$ при $T>2 \mathrm{~K}$ наблюдается переход к неуниверсальному значению $\kappa=0.73 \pm 0.02$, регулярно наблюдающемуся в системах с крупномасштабным примесным потенциалом.

Для переходов $4 \rightarrow 5$ и $5 \rightarrow 6$ при $T>3 \mathrm{~K}$ температурные зависимости ширин пиков сопротивления описываются скейлинговым законом с $\kappa=0.63 \pm 0.02$ и $\kappa=0.55 \pm 0.02$ соответственно. Наблюдаемый при понижении температуры переход к линейной зависимости $\Delta B=\alpha T+\beta$ может свидетельствовать о конечной ширине полосы делокализованных состояний при $T \rightarrow 0$ из-за перемешивания и перекрытия уровней Ландау с одинаковыми номерами и противоположно направленными спинами.

Работа выполнена в рамках государственного задания ФАНО России (шифр „Электрон“, г.р. № AАAА-A18-118020190098-5) и проекта № 18-10-2-6 программы ФИ УрО РАН, при поддержке РФФИ: 18-02-00172 (образцы), 18-32-00382 (экспериментальные результаты), 18-02-00192 (теоретическое сопровождение).

Работа выполнена с использованием оборудования Центра коллективного пользования НИЯУ МИФИ „Гетероструктурная СВЧ-электроника и физика широкозонных полупроводников“". Измерения выполнены на оборудовании Центра коллективного пользования ИФМ УрО РАН „Испытательный центр нанотехнологий и передовых материалов“.

\section{Список литературы}

[1] A.M.M. Pruisken. Phys. Rev. Lett., 61, 1297 (1988)

[2] B. Huckestein. Rev. Mod. Phys., 67, 357 (1995).

[3] A.M.M. Pruisken. Int. J. Mod. Phys. B, 24, 1895 (2010).
[4] J.T. Chalker, P.D. Coddington. J. Phys. C: Sol. St. Phys., 21, 2665 (1988).

[5] H.P. Wei, D.C. Tsui, M.A. Paalanen, A.M.M. Pruisken. Phys. Rev. Lett., 61, 1294 (1988).

[6] W. Li, J.S. Xia, C. Vicente, N.S. Sullivan, W. Pan, D.C. Tsui, L.N. Pfeiffer, K.W. West. Phys. Rev. B, 81, 033305 (2010).

[7] W. Li, C.L. Vicente, J.S. Xia, W. Pan, D.C. Tsui, L.N. Pfeiffer, K.W. West. Phys. Rev. Lett., 102, 216801 (2009).

[8] N.Q. Balaban, U. Meirav, I. Bar-Joseph. Phys. Rev. Lett., 81, 4967 (1998).

[9] J. Wakabayashi, M. Yamane, S. Kawaji. J. Phys. Soc. Jpn., 58, 1903 (1989).

[10] S. Koch, R.J. Haug, K. von Klitzing, K. Ploog. Phys. Rev. Lett., 67, 883 (1991).

[11] S.Koch, R.J. Haug, K. von Klitzing, K. Ploog. Phys. Rev. B, 43, 6828 (1991).

[12] D. Shahar, M. Hilke, C.C. Li, D.C. Tsui, S.L. Sondhi, J.E. Cunningham, M. Razeghi. Sol. St. Commun., 107, 19 (1998).

[13] D. Shahar, D.C. Tsui, M. Shayegan, E. Shimshoni, S.L. Sondhi. Phys. Rev. Lett., 79, 479 (1996).

[14] P.T. Coleridge. Sol. St. Commun., 112, 241 (1999).

[15] R.T.F. van Schaijk, A. de Visser, S. Olsthoorn, H.P. Wei, A.M.M. Pruisken. Phys. Rev. Lett., 84, 1567 (2000).

[16] Yu.G. Arapov, G.A. Alshanskii, G.I. Harus, V.N. Neverov, N.G. Shelushinina, M.V. Yakunin, O.A. Kuznetsov. Nanotechnology, 13, 86 (2002).

[17] L. Wang, T. Tu, C. Zhou, Y-J. Zhao, G-C. Guo, G-P. Guo. Mod. Phys. Lett., 27, 1350202 (2013).

[18] Z. Wang, D-H. Lee, X- G. Wen. Phys. Rev. Lett., 72, 2454 (1994).

[19] D.K. Lee, J.T. Chalker. Phys. Rev. Lett., 72, 1510 (1994).

[20] G. Xiong, S.-D. Wang, Q. Niu, Y. Wang, X.C. Xie, D.-C. Tian, X.R. Wang. J. Phys.: Condens. Matter, 18, 2029 (2006).

[21] G. Xiong, S.-D. Wang, Q. Niu, Y. Wang, X.R. Wang. EPL, 82, 47008 (2008).

[22] F.W. VanKeuls, H.W. Jiang, A.J. Dahm. Czech. J. Phys., 46, Suppl. S5, 2467 (1996).

[23] R. Meisels, F. Kuchar, W. Belitsch, B. Kramer. Microelectron. Eng., 47, 23 (1999).

[24] D.G. Polyakov, M.E. Raikh. Phys. Rev. Lett., 75, 1368 (1995).

[25] S.W. Hwang, H.P. Wei, L.V. Engel, D.C. Tsui, A.M.M. Pruisken. Phys. Rev. B, 48, 11416 (1993).

[26] L.W. Engel, D.Shahar, C. Kurdak, D.C. Tsui. Phys. Rev. Lett., 71, 2638 (1993).

[27] H.P. Wei, L.W. Engel, D.C. Tsui. Phys. Rev. B, 50, 14609 (1994).

[28] Y.J. Zhao, T. Tu, X.J. Hao, G.C. Guo, H.W. Jiang, G.P. Guo. Phys. Rev. B, 78, 233301 (2008).

[29] S.V. Gudina, Yu.G. Arapov, A.P. Savelyev, V.N. Neverov, S.M. Podgornykh, N.G. Shelushinina, M.V. Yakunin, K. Rogacki, I.S. Vasil'evskii, A.N. Vinichenko. JMMM, 440, 10 (2017).

[30] I.S. Vasil'evskii, S.S. Pushkarev, M.M. Grekhov, A.N. Vinichenko, D.V. Lavrukhin, O.S. Kolentsova. Semiconductors, 50, 559 (2016).

[31] I.S. Vasil'evskii, G.B. Galiev, E.A. Klimov, A.L. Kvanin, S.S. Pushkarev, M.A. Pushkin. Semiconductors, 45, 1158 (2011).

[32] Yu.G. Arapov, S.V. Gudina, A.S. Klepikova, V.N. Neverov, S.G. Novokshonov, G.I. Kharus, N.G. Shelushinina, M.V. Yakunin. JETP, 117, 144 (2013). 
[33] A.J.M. Giesbers, U. Zeitler, L.A. Ponomarenko, R. Yang, K.S. Novoselov, A.K. Geim, J.C. Maan. Phys. Rev. B, 80, 241411(R) (2009).

[34] T. Khouri, M. Bendias, P. Leubner, C. Brune, H. Buhmann, L.W. Molenkamp, U. Zeitler, N.E. Hussey, S. Wiedmann. Phys. Rev. B, 93, 125308 (2016).

[35] H.P. Wei, S.Y. Lin, D.C. Tsui, A.M.M. Pruisken. Phys. Rev. B, 45, 3926(R) (1992).

[36] W. Li, G.A. Csáthy, D.C. Tsui, L.N. Pfeiffer, K.W. West. Phys. Rev. Lett., 94, 206807 (2005).

[37] B. Karmakar, M.R. Gokhale, A.P. Shah, B.M. Arora, D.T.N. de Lang, A. de Visser, L.A. Ponomarenko, A.M.M. Pruisken. Physica E, 24, 187 (2004).

[38] A.M.M. Pruisken, I.S. Burmistrov. JETP Lett., 87, 220 (2008).

[39] I.S. Burmistrov, S. Bera, F. Evers, I.V. Gornyi, A.D. Mirlin. Ann. Phys., 326, 1457 (2011).

[40] Yu.G. Arapov, S.V. Gudina, V.N. Neverov, S.M. Podgornykh, M.R. Popov, G.I. Harus, N.G. Shelushinina, M.V. Yakunin, N.N. Mikhailov, S.A. Dvoretskiy. Semiconductors, 49, 1545 (2015).

[41] S. Koch, R.J. Haug, K.v. Klitzing, K. Ploog. Semicond. Sci. Technol. 10, 209 (1995).

[42] K.-H. Yoo, H.C. Kwon, J.C. Park. Sol. St. Commun., 92, 821 (1994).

[43] F. Hohls, U. Zeitler, R.J. Haug. Phys. Rev. Lett., 88, 036802 (2002).

[44] N.A. Dodoo-Amoo, K. Saeed, D. Mistry, S.P. Khanna, L. Li, E.N. Linfield, A.G. Davies, J.E. Cunningham. J. Phys.: Condens. Matter, 26, 475801 (2014).

[45] W. Li, G.H. Csathy, D.C. Tsui, L.N. Pfeiffer, K.W. West. Appl. Phys. Lett., 83, 2832 (2003).

[46] A.M.M. Pruisken, C.B. Scoric, M.A. Baranov. Phys. Rev. B, 60, 16838 (1999).

[47] C.-H. Liu, P.-H. Wang, T.-P. Woo, F.-Y. Shih, S.-C. Liou, P.-H. Ho, C.-W. Chen, C.-T. Liang, W.-H. Wang. Phys. Rev. B, 93, 041421(R) (2016).

[48] D.H. Lee, Z. Wang. Phys. Rev. Lett., 76, 4014 (1996).

[49] A.M.M. Pruisken, M.A. Baranov. Europhys. Lett., 31, 543 (1995).

[50] A.M. Finkelstein. Int. J. Mod. Phys. B, 24, 1855 (2010).

[51] Yu.G. Arapov, S.V. Gudina, A.S. Klepikova, V.N. Neverov, G.I. Harus, N.G. Shelushinina, M.V. Yakunin. Low Temp. Phys., 41, 106 (2015).

[52] S.V. Gudina, Yu.G. Arapov, A.P. Saveliev, V.N. Neverov, S.M. Podgornykh, N.G. Shelushinina, M.V. Yakunin, I.S. Vasil'evskii, A.N. Vinichenko (unpublished).

[53] С.В. Гудина, Е.В. Ильченко, В.Н. Неверов, С.М. Подгорных, Н.Г. Шелушинина, М.В. Якунин, Н.Н. Михайлов, С.А. Дворецкий. Тез. докл. ХІІІ Росс. конф. по физике полупроводников (Екатеринбург, 2017) с. 174.

[54] A.L. Efros. Phys. Rev. B, 45, 11354 (1992).

[55] N.R. Cooper, J.T. Chalker. Phys. Rev. B, 484530 (1994).

Редактор А.Н. Смирнов

\section{Non-universal scaling behavior of conductivity peak widths in quantum Hall regime in InGaAs/InAIAs structures}

\author{
S.V. Gudina ${ }^{1}$, Yu.G. Arapov ${ }^{1}$, E.I. Ilchenko ${ }^{1}$, \\ V.N. Neverov ${ }^{1}$, A.P. Savelyev ${ }^{1}$, S.M. Podgornyk $h^{\mathbf{1}, 2}$, \\ N.G. Shelushinina ${ }^{1}$, M.V. Yakunin ${ }^{1,2}$, \\ I.S. Vasil'evskii ${ }^{3}$, A.N. Vinichenko ${ }^{3}$
}

${ }^{1}$ Miheev Institute of Metal Physics of Ural Branch of Russian Academy of Sciences, 620108 Yekaterinburg, Russia

${ }^{2}$ Ural Federal University, 620002 Yekaterinburg, Russia

${ }^{3}$ National Research Nuclear University „MEPhl“, 115409 Moscow, Russia

Abstract For an investigation of the quantum phase transitions in quantum Hall effect regime on $n-\operatorname{In}_{0.9} \mathrm{Ga}_{0.1} \mathrm{As} / \mathrm{In}_{0.81} \mathrm{Al}_{0.19} \mathrm{As}$ structures the longitudinal $\rho_{x x}$ and Hall $\rho_{x y}$ magnetoresistances were measured in magnetic fields up to $12 \mathrm{~T}$ at $T=0.4-30 \mathrm{~K}$. Non-universal scaling behavior of temperature dependence of resistivity peak widths associated with the presence of large-scale random potential and mixing of Landau levels with opposite spin directions was found. 